\title{
Predicting abundance-body size relationships in functional and taxonomic subsets of food webs
}

\author{
T. A. D. Maxwell · S. Jennings
}

Published online: 16 December 2006

(C) Springer-Verlag 2006

\section{Erratum to: Oecologia 150:282-290 \\ DOI 10.1007/s00442-006-0520-2}

In the original published version of this article, the text read:

1. In the Results section

For values of $\Delta \delta^{15} \mathrm{~N}$ from 2.4 to $3.4 \%$, a range that encompasses all values of $\Delta \delta^{15} \mathrm{~N}$ within $1 \mathrm{SD}$ of the mean fractionation reported in the review of Post (2002), the variations in predicted slope within sites were relatively small in relation to confidence limits around observed slopes (Table 2) at all realistic transfer efficiencies.

2. In the Discussion section

However, our sensitivity analyses demonstrated that varying $\Delta \delta^{15} \mathrm{~N}$ from 2.4 to $3.4 \%$, a range that encompassed all values of $\Delta \delta^{15} \mathrm{~N}$ within $1 \mathrm{SD}$ of the mean ...

And the text should read:

1. In the Results section

For values of $\Delta \delta^{15} \mathrm{~N}$ from 2.4 to $4.4 \%$, a range that encompasses all values of $\Delta \delta^{15} \mathrm{~N}$ within $1 \mathrm{SD}$ of the mean fractionation reported in the review of Post (2002), the variations in predicted slope within sites were relatively small in relation to confidence limits around observed slopes (Table 2) at all realistic transfer efficiencies.
2. In the Discussion section

However, our sensitivity analyses demonstrated that varying $\Delta \delta^{15} \mathrm{~N}$ from 2.4 to $4.4 \%$, a range that encompassed all values of $\Delta \delta^{15} \mathrm{~N}$ within $1 \mathrm{SD}$ of the mean ...

The online version of the original article can be found at http://dx.doi.org/10.1007/s00442-006-0520-2.

T. A. D. Maxwell $(\bowtie) \cdot$ S. Jennings

Centre for Environment, Fisheries and Aquaculture Science,

Lowestoft Laboratory, Lowestoft NR33 OHT, UK

e-mail: tracy.maxwell@cefas.co.uk 